hep-th/0403103

\title{
General Definition of Gravitational Tension
}

\author{
Troels Harmark and Niels A. Obers \\ The Niels Bohr Institute \\ Blegdamsvej 17, 2100 Copenhagen Ø, Denmark \\ harmark@nbi.dk, obers@nbi.dk
}

\begin{abstract}
In this note we give a general definition of the gravitational tension in a given asymptotically translationally-invariant spatial direction of a space-time. The tension is defined via the extrinsic curvature in analogy with the Hawking-Horowitz definition of energy. We show the consistency with the ADM tension formulas for asymptotically-flat spacetimes, in particular for Kaluza-Klein black hole solutions. Moreover, we apply the general tension formula to near-extremal branes, constituting a check for non-asymptotically flat space-times.
\end{abstract}




\section{Introduction and summary}

In this note we give a general definition of the gravitational tension in a given asymptotically translationally-invariant spatial direction of a space-time. The definition is general in the sense that while it reduces to the correct tension formulas for asymptotically-flat space-times it can also be applied to non-asymptotically flat space-times. So far, general tension formulas are known for asymptotically-flat space-times [1, 2, 3, 4, but it has only been computed in particular cases of non-asymptotically flat space-times [5, 6, 7, 8].

The general definition of gravitational tension is obtained in Section 2 by generalizing the derivation of the Hawking-Horowitz energy formula [9]. In particular this means that the tension is defined relative to a reference space-time, thus making it a necessary requirement for the applicability of the tension definition to have a sensible choice of reference space-time. The tension formula is derived by applying the Hamiltonian formalism to a foliation of the space-time along the spatial direction in which we want to measure the tension, in analogy with the Hawking-Horowitz energy formula in which the space-time is foliated along the time-direction. ${ }^{1}$

In Section 3 we show that the general tension formula correctly reduces to the known ADM-type expressions [1, 2, 3, 4] for asymptotically-flat space-times. Moreover, following the work of [1, 2, 3, 4, we present a general Smarr formula that holds in this case and gives the first law of thermodynamics, including the work term associated to the tension. We also give the general bounds satisfied by the tension following from the positivity of gravitational tension [10, 11] and the Strong Energy Condition.

As an illustration of the general tension formula we have included two simple examples in Section 4. The first example is that of vacuum solutions of higher-dimensional General Relativity for Kaluza-Klein type space-times, i.e. Minkowski-space times a circle. Of specific interest are static and neutral solutions with event horizons, which we here call Kaluza-Klein black holes. We show that one recovers the tension formula for the circle direction in this case, previously obtained in 3, 4, and used for Kaluza-Klein black holes in [3, 4, 12].

The second example we consider is that of near-extremal branes of type IIA/B String theory and M-theory, which are non-asymptotically flat space-times. We show that the general expression correctly reproduces the known results [5, 8] for the tension along the spatial world-volume directions. For most of these cases the measured tension is negative which is natural since the gravitational tension has a dual interpretation as the pressure of the non-gravitational theory living on the world-volume of the brane.

Since branes and other objects with tensions play a central role in General Relativity, String/M-theory and holographic dualities between gravitational and non-gravitational theories, many other applications of the general gravitational tension formula can be en-

\footnotetext{
${ }^{1}$ Note that the idea of defining the tension using the Hamiltonian formalism for a spatial direction is from 1 where it is used to derive a formula for the tension for asymptotically-flat space-times.
} 
visioned. In a forthcoming work [13, we consider a combination of the two examples mentioned above, namely near-extremal branes on a transverse circle. This will constitute a new application of the definition of gravitational tension given in this note.

Finally, we have included three appendices. In the first one we show how to get the ADM-type tension formula for asymptotically flat space-times from the general tension formula. In the second one we solve the linearized Einstein equations to show the connection between the ADM-type expressions for asymptotically-flat space-times and a second set of expressions that can be derived in this case. The third appendix contains some details of the proof of the Smarr formula.

\section{General definition of gravitational tension}

In Section 2.1 we use the Hamiltonian approach for a foliation of the space-time along a spatial direction to argue for the general definition of gravitational tension for a spacetime. In the process we repeat the derivation of the Hawking-Horowitz formula for energy 9]. In Section 2.2 we summarize the results of Section 2.1 and write down the explicit expression for the definition of gravitational tension.

\subsection{Hamiltonian approach to definition of energy and tension}

In this section we generalize the Hamiltonian approach to defining energy in 9 so that we also get a general definition of the tension. In order to be pedagogical and complete part of the following derivation is a repeat of [9]. See also [14] for the formalism used.

The basic idea in the following is to generalize the Hamiltonian approach of [9] so that we can consider both a foliation of the space-time along a time direction and a spatial direction. While for a time direction we get the Hawking-Horowitz definition of energy we get for a spatial direction instead a general definition of tension. The idea of defining the tension from the Hamiltonian along a spatial direction is from [1] where it is used to get a general definition of the tension for asymptotically-flat space-times.

The Einstein-Hilbert action for a $D$-dimensional Lorentzian manifold $\left(M, g_{\mu \nu}\right)$ with boundary $\partial M$ is

$$
I=\frac{1}{16 \pi G} \int_{M} R+\frac{1}{8 \pi G} \int_{\partial M} K
$$

where $g_{\mu \nu}$ is the metric on $M$. Here $R$ is the Ricci scalar curvature and $K$ is the extrinsic curvature on $\partial M$. We suppress the integration measures here and in the following. We could also add matter terms to the action. However, since they in general only include first order derivatives, these will not be important for defining the energy or tension and we thus ignore them in this discussion.

In the following we shall use (2.1) to define a Hamiltonian on $M$ with respect to a given time-like or space-like foliation of $M$. Subtracting the Hamiltonian on a reference 
space then gives a natural and general definition of the value of the Hamiltonian which we subsequently use to define the energy and tension.

Consider a family of codimension one submanifolds $\left\{\Sigma_{x}\right\}$ of $M$ labeled by the parameter $x \in\left[x_{\min }, x_{\max }\right]$, given such that $\left\{\Sigma_{x}\right\}$ is a foliation of $M$. Consider moreover a vector field $X^{\mu}$ on $M$ satisfying $X^{\mu} \partial_{\mu} x=1$. We can think of $X^{\mu}$ as defining the "flow of $x$ " throughout the space-time. In the following we consider either the case where the family of submanifolds $\left\{\Sigma_{x}\right\}$ all are Euclidean and $X^{\mu}$ is time-like (in which case $x$ can be thought of as a time-coordinate) or the case where $\left\{\Sigma_{x}\right\}$ all are Lorentzian and $X^{\mu}$ is space-like.

Consider now the unit normal $n^{\mu}$ of the submanifold $\Sigma_{x}$, so that $n^{\mu} n_{\mu}=s$ where $s=1$ $(s=-1)$ if $X^{\mu}$ is space-like (time-like). We can then decompose $X^{\mu}$ into parts normal and tangential to $\Sigma_{x}$

$$
X^{\mu}=N n^{\mu}+N^{\mu}
$$

defining the lapse function $N$ and shift vector $N^{\mu}$. The metric on $\Sigma_{x}$ with respect to $n^{\mu}$ is

$$
g_{\mu \nu}^{(x)}=g_{\mu \nu}-s n_{\mu} n_{\nu}
$$

The extrinsic curvature tensor on $\Sigma_{x}$ with respect to $n^{\mu}$ is

$$
K_{\mu \nu}=D_{\mu} n_{\nu}
$$

We assume the foliation $\left\{\Sigma_{x}\right\}$ of $M$ to be such that the boundary $\partial M$ of $M$ consist of three separate pieces: The "initial" boundary $\Sigma_{x_{\min }}$, the "final" boundary $\Sigma_{x_{\max }}$, and the "asymptotic boundary" $\Sigma^{\infty}$, so that

$$
\partial M=\Sigma_{x_{\min }} \cup \Sigma_{x_{\max }} \cup \Sigma^{\infty} .
$$

Let $r^{\mu}$ be a unit normal vector field on $\Sigma^{\infty}$. We assume furthermore the foliation $\left\{\Sigma_{x}\right\}$ of $M$ to be such that $n^{\mu}$ is a tangent on $\Sigma^{\infty}$, i.e. such that $r \cdot n=0$. Defining now the intersections $S_{x}^{\infty}=\Sigma_{x} \cap \Sigma^{\infty}$ we can foliate $\Sigma^{\infty}$ by the family of submanifolds $\left\{S_{x}^{\infty}\right\}$.

Note that the data of the metric $g_{\mu \nu}$ is contained in $\left(g_{\mu \nu}^{(x)}, N, N_{\mu}\right)$ and vice versa. To obtain the Hamiltonian corresponding to the flow of $x$, we now rewrite the action (2.1) in terms of the latter.

Defining $G_{\mu \nu}=R_{\mu \nu}-\frac{1}{2} g_{\mu \nu} R$ we have

$$
\begin{gathered}
R=2 s\left(R_{\mu \nu}-G_{\mu \nu}\right) n^{\mu} n^{\nu}, 2 G_{\mu \nu} n^{\mu} n^{\nu}=-s R^{(x)}+K^{2}-K_{\mu \nu} K^{\mu \nu} \\
R_{\mu \nu} n^{\mu} n^{\nu}=K^{2}-K_{\mu \nu} K^{\mu \nu}-D_{\mu}\left(n^{\mu} D_{\nu} n^{\nu}\right)+D_{\nu}\left(n^{\mu} D_{\mu} n^{\nu}\right)
\end{gathered}
$$

so that

$$
R=R^{(x)}+s\left[K^{2}-K_{\mu \nu} K^{\mu \nu}\right]-2 s\left[D_{\mu}\left(n^{\mu} D_{\nu} n^{\nu}\right)-D_{\nu}\left(n^{\mu} D_{\mu} n^{\nu}\right)\right]
$$


for a given submanifold $\Sigma_{x}$. The last two terms here are boundary terms and combining with the extrinsic curvature term we get the boundary action

$$
8 \pi G I_{\mathrm{bd}}=\int_{\partial M} K-s \int_{M}\left[D_{\mu}\left(n^{\mu} D_{\nu} n^{\nu}\right)-D_{\nu}\left(n^{\mu} D_{\mu} n^{\nu}\right)\right] .
$$

For the contribution on the $\Sigma_{x_{\min }}$ and $\Sigma_{x_{\max }}$ part of $\partial M$ (with normal vectors $-n^{\mu}$ at $\Sigma_{x_{\min }}$ and $n^{\mu}$ at $\left.\Sigma_{x_{\max }}\right)$ we have

$$
-\int_{\Sigma_{x_{\min }}}\left[g^{\mu \nu} D_{\mu} n_{\nu}-s(n \cdot n) D_{\nu} n^{\nu}\right]+\int_{\Sigma_{x_{\max }}}\left[g^{\mu \nu} D_{\mu} n_{\nu}-s(n \cdot n) D_{\nu} n^{\nu}\right]=0 .
$$

Here the last term in (2.9) does not contribute since $n_{\nu} n^{\mu} D_{\mu} n^{\nu}=0$ because $n$ is unit vector.

For the contribution from the boundary at infinity $\Sigma^{\infty}$ (with normal vector $r^{\mu}$ ) we have on the other hand

$$
\int_{\Sigma \infty}\left[g^{\mu \nu} D_{\mu} r_{\nu}+s r_{\nu} n^{\mu} D_{\mu} n^{\nu}\right]
$$

since the second term in (2.9) does not contribute because $r \cdot n=0$. After integrating by parts the second term in this expression we can write this as

$$
\int_{\Sigma^{\infty}}\left[g^{\mu \nu}-s n^{\mu} n^{\nu}\right] D_{\mu} r_{\nu}
$$

We recognize now $g_{\mu \nu}^{(x)}=g_{\mu \nu}-s n_{\mu} n_{\nu}$ as the metric on $\Sigma_{x}$ so that the integrand of (2.12) is nothing but the extrinsic curvature $K^{(D-2)}=\left(g^{(x)}\right)^{\mu \nu} D_{\mu}^{(x)} r_{\nu}$ of the surface $S_{x}^{\infty}$ in $\Sigma_{x}$. Here $D_{\mu}^{(x)}$ is the covariant derivative in $\Sigma_{x}$. We get thus that (2.9) can be written as

$$
8 \pi G I_{\mathrm{bd}}=\int d x N \int_{S_{x}^{\infty}} K^{(D-2)} .
$$

Using the above we can therefore write the action (2.1) as

$$
I=\frac{1}{16 \pi G} \int d x N\left[\int_{\Sigma_{x}}\left(R^{(x)}+s\left[K^{2}-K_{\mu \nu} K^{\mu \nu}\right]\right)+2 \int_{S_{x}^{\infty}} K^{(D-2)}\right] .
$$

We now further rewrite the bulk part of (2.14)

$$
\mathcal{L}_{\text {bulk }}=\sqrt{g^{(x)}}\left(R^{(x)}+s\left[K^{2}-K_{\mu \nu} K^{\mu \nu}\right]\right),
$$

to a form appropriate for extracting the Hamiltonian. For this we introduce the canonical momentum

$$
p^{\mu \nu} \equiv \frac{1}{\sqrt{g^{(x)}}} \pi^{\mu \nu}=\frac{1}{\sqrt{g^{(x)}}} \frac{\partial \mathcal{L}_{\mathrm{bulk}}}{\partial \dot{g}_{\mu \nu}^{(x)}},
$$

with

$$
\dot{g}_{\mu \nu}^{(x)}=\left(g^{(x)}\right)_{\mu}^{\rho}\left(g^{(x)}\right)_{\nu}{ }^{\sigma} \mathcal{L}_{X} g_{\rho \sigma}
$$

and use that the extrinsic curvature $K_{\mu \nu}$ is related to $\dot{g}_{\mu \nu}^{(x)}$ by

$$
K_{\mu \nu}=\frac{1}{2 N}\left[\dot{g}_{\mu \nu}^{(x)}-2 D_{(\mu}^{(x)} N_{\nu)}\right] .
$$


Then we can write

$$
\mathcal{L}_{\text {bulk }}=\sqrt{g^{(x)}}\left(p^{\mu \nu} \dot{g}_{\mu \nu}^{(x)}-\left[-s N \mathcal{H}+N^{\mu} \mathcal{H}_{\mu}\right]-2 D_{\mu}\left(N_{\nu} p^{\mu \nu}\right)\right),
$$

where the Hamiltonian constraints are given by

$$
\mathcal{H}=R^{(x)}+p^{\mu \nu} p_{\mu \nu}-\frac{1}{D-2} p^{2}, \quad \mathcal{H}_{\mu}=-2 D_{\mu} p^{\mu \nu},
$$

with $p=p_{\mu}{ }^{\mu}$. The last term in (2.19) will contribute an extra boundary term giving the action

$$
I=\frac{1}{16 \pi G} \int d x\left[\int_{\Sigma_{x}}\left(p^{\mu \nu} \dot{g}_{\mu \nu}^{(x)}-\left[-s N \mathcal{H}+N^{\mu} \mathcal{H}_{\mu}\right]\right)+2 \int_{S_{x}^{\infty}}\left(N K^{(D-2)}-N^{\nu} p_{\mu \nu} r_{\nu}\right)\right]
$$

From this action we can extract the Hamiltonian

$$
H=\frac{1}{16 \pi G} \int_{\Sigma_{x}}\left[-s N \mathcal{H}+N^{\mu} \mathcal{H}_{\mu}\right]-\frac{1}{8 \pi G} \int_{S_{x}^{\infty}}\left[N K^{(D-2)}-N^{\nu} p_{\mu \nu} r_{\nu}\right]
$$

Now, in order to define the physical Hamiltonian we must choose a reference background. Thus, given the space-time manifold $\left(M, g_{\mu \nu}\right)$ we choose a space-time manifold $\left(M,\left(g_{0}\right)_{\mu \nu}\right)$ as the reference background such that $g_{\mu \nu}=\left(g_{0}\right)_{\mu \nu}$ on the boundary $\Sigma^{\infty} .^{2}$ The requirement that $g_{\mu \nu}$ and $\left(g_{0}\right)_{\mu \nu}$ coincide on $\Sigma^{\infty}$ can be relaxed to an approximate agreement, provided the approximation becomes exact as $\Sigma_{\infty}$ recedes to infinity. We require furthermore that $X^{\mu}$ is a Killing vector on $\left(M,\left(g_{0}\right)_{\mu \nu}\right)$, i.e. that $\mathcal{L}_{X}\left(g_{0}\right)_{\mu \nu}=0$.

Since $\left(M,\left(g_{0}\right)_{\mu \nu}\right)$ is invariant under translations in $x$, the momenta and the constraints vanish, and the physical Hamiltonian $H-H_{0}$ becomes

$$
H-H_{0}=\frac{1}{16 \pi G} \int_{\Sigma_{x}}\left[-s N \mathcal{H}+N^{\mu} \mathcal{H}_{\mu}\right]-\frac{1}{8 \pi G} \int_{S_{x}^{\infty}}\left[N\left(K^{(D-2)}-K_{0}^{(D-2)}\right)-N^{\nu} p_{\mu \nu} r_{\nu}\right],
$$

where $K_{0}^{(D-2)}$ is the extrinsic curvature of $S_{x}^{\infty}$ in the reference space $\left(M,\left(g_{0}\right)_{\mu \nu}\right)$, with the appropriate choice of labelling of slices so that $N_{0}=N$.

We then define the energy or tension associated to translations in $x$ (time or space) as

$$
-\frac{1}{8 \pi G} \int_{S_{x}^{\infty}}\left[N\left(K^{(D-2)}-K_{0}^{(D-2)}\right)-N^{\nu} p_{\mu \nu} r_{\nu}\right]
$$

\subsection{General formulas for energy and tension}

We now write down the general formulas for energy and tension that follow from (2.24) in more detail.

Consider first a time-like $X^{\mu}$, which means that $s=-1$, i.e. the submanifolds $\left\{\Sigma_{x}\right\}$ are Euclidean. Then $x$ is a time, and we can then define the energy/mass for the space-time $\left(M, g_{\mu \nu}\right)$ with respect to the static space-time $\left(M,\left(g_{0}\right)_{\mu \nu}\right)$ as

$$
E=-\frac{1}{8 \pi G} \int_{S_{x}^{\infty}}\left[N\left(K^{(D-2)}-K_{0}^{(D-2)}\right)-N^{\nu} p_{\mu \nu} r_{\nu}\right]
$$

\footnotetext{
${ }^{2}$ If we include matter fields $\phi_{i}$ we also need that $\left(\phi_{0}\right)_{i}=\phi_{i}$ on $\Sigma^{\infty}$.
} 
where $X^{\mu}=N n^{\mu}+N^{\mu}$. Note that $\left(M, g_{\mu \nu}\right)$ is required to be approximately static at infinity in this case. This formula (2.25) is of course the Hawking-Horowitz formula of [9].

Consider instead now a space-like $X^{\mu}$, which means that $s=1$, i.e. that the submanifolds $\left\{\Sigma_{x}\right\}$ are Lorentzian. Then $x$ is a space direction and we can define the tension using (2.24). However, we would like to cancel out the integration over the time. Assuming that the space-time $\left(M, g_{\mu \nu}\right)$ is stationary we can consider some time-function $t \in\left[t_{\min }, t_{\max }\right]$ for $M$ defining a time-interval $\Delta t=t_{\max }-t_{\min }$. Thus, dividing by the time interval $\Delta t$ will cancel the integration over the time-function $t$, and we define therefore the tension as

$$
\mathcal{T}=-\frac{1}{\Delta t} \frac{1}{8 \pi G} \int_{S_{x}^{\infty}}\left[F\left(K^{(D-2)}-K_{0}^{(D-2)}\right)-F^{\nu} p_{\mu \nu} r_{\nu}\right]
$$

where we have used the notation $X^{\mu}=F n^{\mu}+F^{\mu}$. Note that $\left(M, g_{\mu \nu}\right)$ is required to be approximately translationally invariant along $x$ at infinity in this case. The tension $\mathcal{T}$ in (2.26) is the tension along the $x$ direction.

\section{$3 \quad$ Asymptotically-flat space-times}

In this section we first explain that the general results (2.25), (2.26) reduce to the known ADM-type formulas for mass and tension in asymptotically-flat space-times. We then present in Section 3.2 the general Smarr formula and the first law of thermodynamics.

\subsection{Total mass and tension for asymptotically-flat space-times}

In Ref. 9] it was shown that the expression (2.25) for the mass correctly reduces to the ADM mass for asymptotically-flat space-times [15]

$$
M=\frac{1}{16 \pi G} \int_{S_{t}^{\infty}} d S^{m}\left(\partial^{n} h_{m n}-\delta^{n l} \partial_{m} h_{n l}\right)
$$

Here $g_{m n}^{(t)}=\delta_{m n}+h_{m n}$ is the $(D-1)$-dimensional metric as defined in (2.3) when foliating the space-time along the time $t$. We assume for simplicity here that the background metric is $\delta_{m n}$. The indices $m, n, l$ run over the $D-1$ directions perpendicular to $t$.

In Appendix $\mathrm{A}$ we show that for an asymptotically-flat space-time the general tension formula (2.26) reduces to the ADM-type formula

$$
\mathcal{T}=\frac{1}{16 \pi G} \int_{\hat{S}_{z}^{\infty}} d S^{m}\left(\partial^{n} h_{m n}-\eta^{n l} \partial_{m} h_{n l}\right)
$$

Here $g_{m n}^{(z)}=\eta_{m n}+h_{m n}$ is the $(D-1)$-dimensional metric as defined in (2.3) when foliating the space-time along the spatial direction $z$. We assume for simplicity here that the background metric is $\eta_{m n}=\operatorname{diag}(-1,1, \ldots, 1)$. The indices $m, n, l$ run over the $D-1$ directions perpendicular to $z$. Note that in going from (2.26) to (3.2) we have cancelled the $\Delta t$ factor by integrating over the time at infinity $\int d t=\Delta t$, so that the remaining integration is over $\hat{S}_{z}^{\infty}=S_{z}^{\infty} / \Delta t$. The tension formula (3.2) agrees with the one in [1, 2]. 
The equations above provide a nice set of covariant expressions for the mass and tension(s) in asymptotically-flat space-times with one (or more) space-like isometries at infinity. However, by solving Einstein equations at infinity where the gravitational field is weak and using the principle of equivalent sources ${ }^{3}$ it is possible to reduce to even simpler expressions. The derivation, which builds on [2, 3, 4] is given in Appendix B]

The result is derived for the case of a $D$-dimensional space-time with coordinates $t$, $z^{a}, a=1, \ldots, k, x^{i}, i=1, \ldots, D-k-1$ and $r^{2}=\sum_{i=1}^{D-k-1}\left(x^{i}\right)^{2}$ is the radial coordinate in the transverse space. Beyond the time translation Killing vector $\partial / \partial t$, we assume in addition that $\partial / \partial z_{a}$ are asymptotic Killing vectors. We consider each of the directions $z^{a}$ to be compactified on a circle with circumference $L_{a}$. Finally, the space is taken to be asymptotically flat, so that the spatial part of the metric at infinity is $\mathbb{R}^{D-k-1} \times \mathbb{T}^{k}$, where $\mathbb{T}^{k} \equiv\left(S^{1}\right)^{k}$ is a rectangular $k$-torus with volume $V_{k}=\prod_{a=1}^{k} L_{a}$.

The mass $M$ and tensions $\mathcal{T}_{a}$ in each of the compact directions are then given by ${ }^{4}$

$$
\begin{gathered}
M=\frac{V_{k} \Omega_{D-k-2}}{16 \pi G}\left[(D-k-2) c_{t}-\sum_{a} c_{a}\right], \\
\mathcal{T}_{a}=\frac{V_{k} \Omega_{D-k-2}}{16 \pi G L_{a}}\left[c_{t}-(D-k-2) c_{a}-\sum_{b \neq a} c_{a}\right], a=1, \ldots, k,
\end{gathered}
$$

where $c_{t}$ and $c_{a}$ are the leading corrections of the metric at infinity

$$
g_{t t}=-1+\frac{c_{t}}{r^{D-k-3}}, \quad g_{a a}=1+\frac{c_{a}}{r^{D-k-3}}, \quad a=1, \ldots, k
$$

These equations enable one to measure the mass (3.1) and tension (3.2) for the class of space-times defined above in terms of the asymptotics of the metric.

It is important to realize that not all values of $M$ and $\mathcal{T}_{a}$ correspond to physically reasonable matter. Just like we demand that $M \geq 0$, we also have bounds on the tensions $\mathcal{T}_{a}$. In the general framework of this section we have the bounds

$$
M \geq \frac{1}{D-3} \sum_{a} L_{a} \mathcal{T}_{a}, \quad \mathcal{T}_{a} \geq 0
$$

The first bound follows from the Strong Energy Condition which basically states that any physically sensible source for the gravitational field can not be repulsive. In terms of the energy-momentum tensor it is the statement that $T_{00}+\frac{1}{D-2} T_{\mu}^{\mu} \geq 0$. Integrating this relation over the space-like directions and using (B.8), (3.8) then gives the stated result.

The bound $\mathcal{T}_{a} \geq 0$ (for a given $a$ ) corresponds instead to the statement that the tension can not be negative, as proven in [10, 11].

\footnotetext{
${ }^{3}$ The principle of equivalent sources is the principle that any source of gravitation affecting the asymptotic region the same way should also have the same values for the physical parameters associated with the sources of the gravitational field.

${ }^{4}$ Here $\Omega_{l}=2 \pi^{\frac{l+1}{2}} / \Gamma\left(\frac{l+1}{2}\right)$ is the volume of the unit $l$-sphere.
} 


\subsection{Smarr formula and first law of thermodynamics}

We can push our general considerations further by splitting up the energy-momentum tensor as the sum

$$
T_{\mu \nu}=T_{\mu \nu}^{\mathrm{gr}}+T_{\mu \nu}^{\mathrm{mat}}
$$

where $T_{\mu \nu}^{\mathrm{gr}}$ is the energy-momentum tensor of the gravitational field relative to the $D$ dimensional flat background and $T_{\mu \nu}^{\text {mat }}$ the contribution of additional matter, such as scalar fields or $p$-form gauge potentials (see also Appendix $\underline{\mathrm{B}}$ ).

Using now the principle of equivalent sources, we assume in the following that our space-time is everywhere nearly flat. We can then write the mass $M$ and tension $\mathcal{T}_{a}$ as

$$
M=\int d^{D-1} x T_{00}, \quad \mathcal{T}_{a}=-\frac{1}{L_{a}} \int d^{D-1} x T_{a a}, \quad a=1, \ldots, k .
$$

where $L_{a}$ is the size of the $a^{\prime}$ th direction.

Using instead the matter energy-momentum tensor $T_{\mu \nu}^{\text {mat }}$ we can write the matter contributions to the mass and tension denoted by $M^{\text {mat }}$ and $\mathcal{T}_{a}^{\text {mat }}$ respectively, as

$$
M^{\mathrm{mat}}=\int d^{D-1} x T_{00}^{\mathrm{mat}}, \mathcal{T}_{a}^{\mathrm{mat}}=-\frac{1}{L_{a}} \int d^{D-1} x T_{a a}^{\mathrm{mat}}, a=1, \ldots, k .
$$

Consider now again the class of space-times for which (3.3), (3.4) was derived, and assume in addition the presence of a single connected event horizon. Then, following [2, 3, 4, it is possible to derive a generalized Smarr formula. For completeness, the derivation is given in Appendix C] Here we present the general Smarr formula (C.7) in the simple form

$$
(D-2) T S=\left(D-3-\sum_{a} n_{a}\right)\left(M-M^{\mathrm{mat}}\right),
$$

where we have introduced the relative tensions

$$
n_{a} \equiv \frac{L_{a}\left(\mathcal{T}_{a}-\mathcal{T}_{a}^{\text {mat }}\right)}{M-M^{\text {mat }}}, a=1, \ldots, k
$$

generalizing the relative tensions introduced in [3..$^{5}$ Note that (3.10), (3.11) depend only on the gravitational contribution to the mass and tensions.

We also give the generalized first law of thermodynamics for this class of space-times,

$$
\delta M=T \delta S+\sum_{a} \mathcal{T}_{a}^{\mathrm{eff}} \delta L_{a}
$$

showing that the effective tension in each compact direction

$$
\mathcal{T}_{a}^{\mathrm{eff}}=\mathcal{T}_{a}-\mathcal{T}_{a}^{\mathrm{mat}}
$$

\footnotetext{
${ }^{5}$ Note that the relative tension $n_{a}$ along a direction was denoted relative binding energy in [3] since the tension also can be viewed as a binding energy along the particular direction. Here we adopt a different nomenclature since it is conventional to denote the quantity measured in (2.26) as the tension.
} 
is governed by the gravitational part of the tension. The first law can be for example obtained by combining the Smarr formula (3.10), with scaling relations and using Euler's theorem [2]. It also correctly reduces to the first law obtained in [3, 4] for the case of neutral black objects on the cylinder.

Finally, we note that while we still impose the bounds (3.6) on the tensions $\mathcal{T}_{a}$, we can in fact now use the above to obtain stronger bounds on our physical variables. This is because the energy-momentum tensor in (3.7) is a sum of a gravitational part and a matter part and we require these bounds to hold independently for both of the two contributions. The reason for this is that we expect both the matter part and the gravitational part to behave in a physically sensible fashion. Since the matter usually obeys the bounds by construction we can extract some stronger bounds on our physical variables by considering the gravitational part by itself. The bounds on the gravitational part are $(D-3)(M-$ $\left.M^{\text {mat }}\right) \leq \sum_{a} L_{a}\left(\mathcal{T}_{a}-\mathcal{T}_{a}^{\text {mat }}\right)$, and $\mathcal{T}_{a}-\mathcal{T}_{a}^{\text {mat }} \geq 0$. Using the definition (3.11) we can write these as

$$
\sum_{a} n_{a} \leq D-3, \quad n_{a} \geq 0
$$

As stated above, these bounds are stronger than the bounds (3.6).

\section{Examples}

In this section we present two simple examples that illustrate the general definition of gravitational tension.

\subsection{Kaluza-Klein black holes}

As the first example we consider vacuum solutions of higher-dimensional General Relativity (i.e. pure gravity) for the Kaluza-Klein type space-times $\mathcal{M}^{d} \times S^{1}$ with $d \geq 4$ where $\mathcal{M}^{d}$ is the $d$-dimensional Minkowski space-time. More precisely, we consider all static vacuum solutions that asymptote to $\mathcal{M}^{d} \times S^{1}$ and that have an event horizon. We call these solutions Kaluza-Klein black holes here.

Define the Cartesian coordinates for $\mathcal{M}^{d}$ as $t, x^{1}, \ldots, x^{d-1}$ along with the radius $r=$ $\sqrt{\left(x^{1}\right)^{2}+\cdots+\left(x^{d-1}\right)^{2}}$. Let moreover $z$ be the coordinate of the $S^{1}$ with period $L$. Since the Kaluza-Klein black holes are asymptotically flat, we may use the expressions (3.3)(3.5) to find the mass $M$ and tension $\mathcal{T}$ along the compact $z$-direction. It then easily follows from these equations that for any given Kaluza-Klein black hole solution we can write [3, 4

$$
M=\frac{\Omega_{d-2} L}{16 \pi G}\left[(d-2) c_{t}-c_{z}\right], \quad \mathcal{T}=\frac{\Omega_{d-2}}{16 \pi G}\left[c_{t}-(d-2) c_{z}\right],
$$

where $c_{t}, c_{z}$ are read off from the metric components

$$
g_{t t}=-1+\frac{c_{t}}{r^{d-3}}, \quad g_{z z}=1+\frac{c_{z}}{r^{d-3}},
$$


for $r \rightarrow \infty$.

We also immediately recover from (3.10), (3.12) the Smarr relation and first law of thermodynamics in this case [3, 4]

$$
(d-1) T S=(d-2-n) M, \quad \delta M=T \delta S+\mathcal{T} \delta L,
$$

where $n=L \mathcal{T} / M$ is the relative tension. Finally, the general relation (3.14) implies the bounds $\underline{3}$

$$
0 \leq n \leq d-2
$$

on the relative tension.

\section{$4.2 \quad$ Near-extremal $p$-branes}

The second example we consider is that of near-extremal $p$-branes of type IIA/B String theory and M-theory. As these branes are not asymptotically flat, this provides a nice application of the general expression (2.26) for gravitational tension.

The near extremal $p$-brane solution in $D=1+p+d$ dimensions has the form

$$
\begin{gathered}
d s^{2}=\hat{H}^{-\frac{d-2}{D-2}}\left(-f d t^{2}+\sum_{k=1}^{p}\left(d u^{k}\right)^{2}+\hat{H}\left[f^{-1} d r^{2}+r^{2} d \Omega_{d-1}^{2}\right]\right), \\
e^{2 \phi}=\hat{H}^{a}, \quad A_{(p+1)}=\hat{H}^{-1} d t \wedge d u^{1} \wedge \cdots \wedge d u^{p}, \quad f=1-\frac{r_{0}^{d-2}}{r^{d-2}}, \quad \hat{H}=\frac{\hat{h}_{d}}{r^{d-2}},
\end{gathered}
$$

where the metric is written in the Einstein frame. Here $\phi$ is the dilaton (for $D=10$ ), $A_{(p+1)}$ the gauge potential and $a$ is a number determining the coupling of the $A_{(p+1)}$ gauge potential to the dilaton. The background asymptotes to the near-horizon limit of the $1 / 2$ BPS extremal $p$-brane of String/M-theory corresponding to (4.5)-(4.6) with $r_{0}=0$, which is taken as the reference background when computing the energy and tension.

The relevant tensions in this case are those corresponding to the $p$ spatial world-volume directions which we take to be compactified on equal size circles of circumference $L$, so that the total volume $V=L^{p}$. Therefore the tension $\mathcal{T}_{k}=\mathcal{T}$ is the same in each direction $u^{k}, k=1, \ldots, p$.

Although the Hawking-Horowitz energy computation is well known for these branes we present for clarity the computation of energy $E$ and tension $\mathcal{T}$ in parallel below. Note that as we are using the extremal $p$-brane as the reference background, the expressions (2.25), (2.26) are of course the energy and tension above extremality. To compute these quantities we first recall that the extrinsic curvature $K^{(D-2)}$ can for our purposes be written as

$$
K^{(D-2)}=\frac{1}{\sqrt{g_{r r}}} \partial_{r} \log \sqrt{\left|g^{D-2}\right|} .
$$

Here $g^{D-2}$ is the determinant of the metric obtained from (4.5) by omitting $t, r$ when computing $E$ and omitting $u^{k}, r$ (for a particular $k$ ) when computing $\mathcal{T}$. We denote the corresponding expressions by $K_{t}$ and $K_{u}$ respectively. 
It is then not difficult to use (4.7) for the metric (4.5) and compute

$$
\begin{gathered}
K_{t}=\sqrt{f\left(r_{m}\right)} \hat{H}\left(r_{m}\right)^{-\frac{p+1}{2(D-2)}} \frac{d}{2 r_{m}}, \\
K_{u}=\sqrt{f\left(r_{m}\right)} \hat{H}\left(r_{m}\right)^{-\frac{p+1}{2(D-2)}}\left[\frac{d}{2}+\frac{d-2}{2}\left(\frac{r_{0}}{r_{m}}\right)^{d-2}\right] \frac{1}{r_{m}},
\end{gathered}
$$

where we used $r_{m} \gg r_{0}$ since $r_{m}$ is sent to infinity in the end. The corresponding expressions for the extrinsic curvature of the reference space in both cases are obtained from (4.8), (4.9) by setting $r_{0}=0$ and substituting $r_{m}$ with $r_{\text {eff }}$. Here $r_{\text {eff }}$ is the radius corresponding to $r_{m}$ for the reference space, defined so that the metrics for the $(D-2)$ dimensional subspaces are equal. The relation between $r_{m}$ and $r_{\text {eff }}$ is then obtained by imposing that the radius of the $S^{d-1}$ in the brane space-time is equal to that of the radius of the $S^{d-1}$ in the reference space. In the present case, this simply means that $r_{\text {eff }}=r_{m}$ to leading order. Finally, we need for each case the lapse functions and integration measures: $N=\left(g_{t t}^{(0)}\right)^{1 / 2}=\hat{H}^{-\frac{d-2}{2(D-2)}}, \sqrt{h}=\sqrt{\hat{H}} r_{m}^{d-1}$ for the energy and $F=\left(g_{z z}^{(0)}\right)^{1 / 2}=\hat{H}^{-\frac{d-2}{2(D-2)}}$, $\sqrt{h}=\sqrt{\hat{H}} r_{m}^{d-1}$ for the tension.

Using all this in (2.25), (2.26) we have the final results

$$
E=\frac{d}{2} \frac{V \Omega_{d-1}}{16 \pi G} r_{0}^{d-2}, L \mathcal{T}=-\frac{d-4}{2} \frac{V \Omega_{d-1}}{16 \pi G} r_{0}^{d-2},
$$

for the energy and spatial world-volume tension of a near-extremal $p$-brane. Note that the quantity $-E+p L \mathcal{T}$ is the trace of the (integrated) energy-momentum tensor on the brane, which is zero only for the non-dilatonic (D3, M2, M5) branes. We remark that the tension was calculated via a different method in Refs. [5, [8] (see also e.g. Refs. [6, 7]).

It is actually more natural to use instead of tension the pressure given by

$$
P=-\frac{L \mathcal{T}}{V}=\frac{d-4}{2} \frac{\Omega_{d-1}}{16 \pi G} r_{0}^{d-2} .
$$

The pressure is positive for $d>4$, which includes the near-extremal D3, M2 and M5 brane. In these cases, we can interpret the pressure directly in terms of the massless excitations of the corresponding field theory at finite temperature.

To consider the thermodynamics of the near-extremal $p$-brane we use the well-known results for temperature and entropy

$$
T=\frac{d-2}{4 \pi} \hat{h}_{d}^{-\frac{d-2}{2}} r_{0}^{\frac{d-4}{2}}, \quad S=\frac{V \Omega_{d-1}}{4 G} \hat{h}_{d}^{\frac{d-2}{2}} r_{0}^{\frac{d}{2}} .
$$

It is then easy to show that the tension is directly related to the Helmholtz free energy

$$
L \mathcal{T}=F, \quad F=E-T S
$$

Together with the identification of the pressure (4.11) this implies that the Gibbs free energy vanishes for near-extremal $p$-branes

$$
G=0, \quad G=E-T S+P V .
$$


It is actually not difficult to verify that any conformally invariant theory in $p+1$ dimensions has $G=0$. Here, however, we see that it holds for all near-extremal $p$-brane theories, regardless whether they are conformal or not. Finally, we note that it is a simple task to explicitly check that the first law $\delta E=T \delta S-P \delta V$ is satisfied using the pressure in (4.11).

\section{Acknowledgments}

We thank Simon Ross for useful discussions.

\section{A Reduction to ADM-type formula}

We show in this appendix how the general tension formula (2.26) reduces to the ADM-type formula (3.2) for asymptotically-flat space-times. Write $g_{m n}^{(z)}=\eta_{m n}+h_{m n}$ as the metric on the $(D-1)$-dimensional subspace transverse to the $z$-direction. Since the reference space-time is flat we can choose the metric of the reference space-time as $\eta_{m n}$. This means that $h_{m n}$ should be zero on $S_{z}^{\infty}$ for the tangential directions.

We now first consider the case of $S_{z}^{\infty}$ being a hyper-cube. In the flat reference background each side of the hyper-cube is defined by one of the $2 D-2$ relations $x^{i}= \pm \alpha$, $i=1, \ldots, D-1$. $\alpha$ is then sent to infinity in the end. Then (2.26) becomes

$$
\mathcal{T}=-\frac{1}{\Delta t} \frac{1}{8 \pi G} \int_{S_{z}^{\infty}} d^{D-2} x\left(K^{(D-2)}-K_{0}^{(D-2)}\right) .
$$

We focus below without loss of generality on the side $x^{1}=\alpha$. For the reference space-time the unit normal vector is simply $\left(r_{0}\right)^{m}=\delta_{m 1}$. For the asymptotically-flat space-time it is instead $r^{m}=\delta_{m 1}-\frac{1}{2} \eta^{m n} h_{n 1} \cdot{ }^{6}$ We have then

$$
K^{(D-2)}=D_{m}^{(z)} r^{m}=\partial_{m} r^{m}+\Gamma_{m n}^{m} r^{n}=\frac{1}{2}\left[-\partial^{n} h_{n m}+\eta^{n l}\left(\partial_{m} h_{n l}\right)\right] r^{m}
$$

where we used that $\partial_{m} r^{m}=-\frac{1}{2} \partial^{n} h_{n 1}=-\frac{1}{2} \partial^{n} h_{n m} r^{m}$ and that $\Gamma_{m n}^{m}=\frac{1}{2} \eta^{m l}\left(\partial_{n} h_{m l}\right)$ to first order in $h_{m n}$. Since $K_{0}^{(D-2)}=\partial_{m}\left(r_{0}\right)^{m}=0$ we get therefore

$$
K^{(D-2)}-K_{0}^{(D-2)}=\frac{1}{2}\left[-\partial^{n} h_{n m}+\eta^{n l}\left(\partial_{m} h_{n l}\right)\right] r^{m} .
$$

Eq. (A.3) clearly holds for all the sides of the hyper-cube. Therefore, (A.1) gives

$$
\mathcal{T}=\frac{1}{\Delta t} \frac{1}{16 \pi G} \int_{S_{z}^{\infty}} d^{D-2} x\left(\partial^{n} h_{n m}-\eta^{n l} \partial_{m} h_{n l}\right) r^{m}
$$

\footnotetext{
${ }^{6}$ This is because $x^{1}=\alpha$ is not the equation for the hypersurface in the asymptotically-flat space-time since the metric is not the Minkowski metric. To find the normal vector notice that we have the inverse Vielbeins $e_{a}^{m}=\delta_{a}^{m}-\frac{1}{2} \eta^{m n} h_{n a}$ so that $g^{m n}=\eta^{a b} e_{a}^{m} e_{b}^{n}$. From the fact that the Vielbeins precisely relate a Minkowski metric to the asymptotically flat metric, we get then that $r^{m}=e_{a}^{m}\left(r_{0}\right)^{a}=e_{1}^{m}=\delta_{m 1}-\frac{1}{2} \eta^{m n} h_{n 1}$.
} 
which clearly is the same as (3.2). One can now generalize to other hypersurfaces $S_{z}^{\infty}$. First we can generalize to surfaces build up by rectangular surfaces of the type $x^{m}=$ constant. For a general hypersurface $S_{z}^{\infty}$ we can then find surfaces built out of rectangular surfaces that are arbitrarily close to $S_{z}^{\infty}$, thus proving that it works for general surfaces also.

\section{B Mass and tension from linearized Einstein equation}

In this appendix we solve the linearized Einstein equations for a static distribution of matter localized on the $\mathbb{R}^{D-k-1}$ times a $k$-torus $\mathbb{T}^{k}$. The derivation builds on that of Refs. 2, 3, 4].

We first define for any energy-momentum tensor $T_{\mu \nu}$ the tensor

$$
S_{\mu \nu} \equiv T_{\mu \nu}-\frac{1}{D-2} \eta_{\mu \nu} T, \quad T=T_{\mu}^{\mu}
$$

Then, by eliminating the Ricci scalar $R$, the Einstein equations with the matter energymomentum tensor $T_{\mu \nu}^{\text {mat }}$ can be written in the form

$$
R_{\mu \nu}=8 \pi G S_{\mu \nu}^{\mathrm{mat}}
$$

where $S_{\mu \nu}^{\text {mat }}$ is related to $T_{\mu \nu}^{\text {mat }}$ via (B.1). In the linearized approximation $g_{\mu \nu}=\eta_{\mu \nu}+h_{\mu \nu}$ we have that

$$
R_{\mu \nu}=R_{\mu \nu}^{(1)}-8 \pi G S_{\mu \nu}^{\mathrm{gr}}
$$

where

$$
R_{\mu \nu}^{(1)}=-\frac{1}{2}\left[\square h_{\mu \nu}+\left(h_{\lambda}^{\lambda}\right)_{, \mu \nu}-\left(h_{\mu}{ }^{\lambda}\right)_{, \nu \lambda}-\left(h_{\nu}{ }^{\lambda}\right)_{, \mu \lambda}\right],
$$

and $S_{\mu \nu}^{\mathrm{gr}}$ determined via (B.1) by the energy-momentum tensor contribution $T_{\mu \nu}^{\mathrm{gr}}$ of the gravitational field relative to the $D$-dimensional Minkowski background. Note that $S_{\mu \nu}^{\mathrm{gr}}$ collects all higher-order terms of $R_{\mu \nu}$. In the weak-field approximation the Einstein equations thus reduce to the system

$$
\square h_{\mu \nu}+\left(h_{\lambda}^{\lambda}\right)_{, \mu \nu}-\left(h_{\mu}{ }^{\lambda}\right)_{, \nu \lambda}-\left(h_{\nu}{ }^{\lambda}\right)_{, \mu \lambda}=-16 \pi G S_{\mu \nu}, \quad S_{\mu \nu}=S_{\mu \nu}^{\mathrm{gr}}+S_{\mu \nu}^{\mathrm{mat}},
$$

and $S_{\mu \nu}$ depends on the total energy-momentum tensor, given by the sum of the gravitational and matter part, $T_{\mu \nu}=T_{\mu \nu}^{\mathrm{gr}}+T_{\mu \nu}^{\mathrm{mat}}$.

Consider now a $D$-dimensional space-time with $k$ compact directions $z^{a}, a=1, \ldots, k$ of periods $L_{a}$, and $D-k-1$ transverse directions $x^{i}, i=1, \ldots, D-k-1$. The spatial part is thus of the from $\mathbb{R}^{D-k-1} \times \mathbb{T}^{k}$, where $\mathbb{T}^{k}=\left(S^{1}\right)^{k}$ is a rectangular $k$-torus. The flat metric thus reads

$$
d s^{2}=-d t^{2}+\sum_{a=1}^{k}\left(d z^{a}\right)^{2}+\sum_{i=1}^{D-k-1}\left(d x^{i}\right)^{2}=-d t^{2}+\sum_{a=1}^{k}\left(d z^{a}\right)^{2}+d r^{2}+r^{2} d \Omega_{D-k-2}^{2}
$$

where we have defined $r^{2}=\sum_{i=1}^{D-k-1}\left(x^{i}\right)^{2}$. 
We consider a static distribution of matter localized on $\mathbb{R}^{D-k-1}$, and assume a diagonal energy-momentum tensor $T_{\mu \nu}$ with non-zero components

$$
T_{00}, T_{a a}, T_{i i}
$$

Here $T_{00}$ is a function of $\left(z^{a}, x^{i}\right)$, while a given $T_{a a}$ depends on all these coordinates except $z^{a}$, because of energy-momentum conservation. We also assume that the resulting spacetime metric is asymptotic to the flat metric (B.6), so that $T_{\mu \nu}$ vanishes in the limit $r \rightarrow \infty$ (sufficiently rapid). Now, energy-momentum conservation implies $\sum_{i} \partial_{i} T_{i j}=0$ so that we can use partial integration to write

$$
\int d^{D-1} x T_{i i}=\int_{S_{\infty}} d S_{j} x^{i} T_{i j}=0
$$

where the first step uses $T_{i i}=\partial_{j}\left(x^{i} T_{i j}\right)$ along with Gauss law $\left(S_{\infty}=S^{D-k-2} \times \mathbb{T}^{k}\right)$ and in the last step we used the vanishing of $T_{i j}$ at spatial infinity in the transverse space. As a consequence of $(\mathbf{B} .8)$ we find in particular that the bulk integrals $\int d^{D-1} x T_{i i}=0$ for the non-zero components $T_{i i}$ in (B.7).

To obtain the relation between the total energy $M$ and tensions $\mathcal{T}_{a}$ in the compact directions defined by (3.8) and the asymptotics of the metric, we now solve the linearized Einstein equations (B.5) for the energy-momentum tensor (B.7). We use the fact that the metric perturbation $h_{\mu \nu}$ is time-independent, along with the fact that it is asymptotically diagonal. Then (B.5) reduce to the set of equations

$$
\begin{array}{r}
\nabla^{2} h_{00}=-16 \pi G S_{00}, \quad \nabla^{2} h_{a a}=-16 \pi G S_{a a}, \quad a=1, \ldots, k, \\
-2 \sum_{i, j=1}^{D-k-1}\left(h_{i j, i j}-h_{i i, j j}\right)-\nabla^{2} h_{00}+\sum_{a} \nabla^{2} h_{a a}=-16 \pi G \sum_{i=1}^{D-k-1} S_{i i},
\end{array}
$$

where $\nabla^{2}$ is the Laplacian operator on $\mathbb{R}^{D-k-1} \times \mathbb{T}^{k}$.

We focus first on the $1+k$ equations in (B.9). Using the definitions for the mass $M$ and binding energies $\mathcal{T}_{a}$ in (3.8), (B.8) as well as (B.1) and (B.8), these are easily solved to leading order yielding

$$
\begin{array}{r}
M=-\frac{1}{D-k-3} \frac{1}{16 \pi G} \int_{S_{\infty}} d S_{i}\left[(D-k-2) h_{00, i}-\sum_{a} h_{a a, i}\right] \\
L_{a} \mathcal{T}_{a}=-\frac{1}{D-k-3} \frac{1}{16 \pi G} \int_{S_{\infty}} d S_{i}\left[h_{00, i}-(D-k-2) h_{a a, i}-\sum_{b \neq a} h_{b b, i}\right] .
\end{array}
$$

These are surface integrals over $S_{\infty}$, which is the $(D-k-2)$-sphere at infinity times the $k$-torus $\mathbb{T}^{k}$. From these we obtain the expressions (3.3), (3.4) in terms of the leading corrections (3.5) of the metric at infinity.

It remains to show that $M$ and $\mathcal{T}_{a}$ in (B.11), (B.12) (and hence the definitions in (3.8), (B.8)) actually agree with the ADM formulae (3.1), (3.2) obtained for the asymptotically 
flat case from the general Hamiltonian treatment. For the case at hand, these can be written as

$$
\begin{gathered}
M^{(\mathrm{ADM})}=\frac{1}{16 \pi G} \int_{S_{\infty}} d S_{i}\left[h_{i j, j}-h_{j j, i}-h_{a a, i}\right], \\
L_{a} \mathcal{T}_{a}^{(\mathrm{ADM})}=\frac{1}{16 \pi G} \int_{S_{\infty}} d S_{i}\left[h_{i j, j}-h_{j j, i}+h_{00, i}-\sum_{b \neq a} h_{b b, i}\right],
\end{gathered}
$$

where we remind the reader that repeated indices are summed over. To this end, we use the linearized Einstein equations (B.10) in the transverse space. Using Eq. (B.9) we can rewrite this relation as

$$
\frac{1}{16 \pi G}\left(h_{i j, i j}-h_{i i, j j}-h_{a a, i i}\right)=\frac{1}{2}\left(S_{00}+S_{a a}+S_{i i}\right) .
$$

Integrating this equation over all spatial dimensions, we see that the left side is the ADM mass formula (B.13). On the other hand, for the right side we may use (B.1), (3.8) and (B.8) to show that $\frac{1}{2} \int d^{D-1} x\left(S_{00}+S_{a a}+S_{i i}\right)=M$. We thus conclude that the relation (B.15) tells us that $M=M_{\mathrm{ADM}}$. Turning to the ADM tension (B.14), rearranging terms this can be written as

$$
\begin{aligned}
L_{a} \mathcal{T}_{a}^{(\mathrm{ADM})} & =\frac{1}{16 \pi G} \int d S_{i}\left[\partial_{i}\left(h_{00}+h_{a a}\right)+\left(h_{i j, j}-h_{j j, i}-h_{b b, i}\right)\right] \\
& =\left(-M+L_{a} \mathcal{T}_{a}\right)+M_{\mathrm{ADM}}=L_{a} \mathcal{T}_{a}
\end{aligned}
$$

where we used (B.11), (B.12), (B.13) in the second step, and in the last step the fact that we already showed $M=M_{\mathrm{ADM}}$. Thus the definitions of the tensions also agree, and we can omit the label "ADM".

\section{Proof of generalized Smarr formula}

To derive the Smarr formula we consider the Komar integral

$$
I_{S}=-\frac{1}{16 \pi G} \int_{S} d S_{\mu \nu} D^{\mu} \xi^{\nu}
$$

where $S$ is a $(D-2)$-dimensional hypersurface and $\xi$ is a Killing vector for the metric. Consider now a static solution on $\mathbb{R}^{D-k-1} \times \mathbb{T}^{k}$ with an event horizon. Consider furthermore a certain time $t=t_{0}$. Define $S_{h}$ to be the null-surface of the event horizon at $t=t_{0}$. We also choose a $(D-2)$ dimensional surface at $r=\infty$ for $t=t_{0}$, which we call $S_{\infty}$, so that essentially $S_{\infty}=S^{D-k-2} \times \mathbb{T}^{k}$. By Gauss theorem we have

$$
I_{S_{h}}-I_{S_{\infty}}=\frac{1}{16 \pi G_{N}}\left(\int_{S_{\infty}} d S_{\mu \nu} D^{\mu} \xi^{\nu}-\int_{S_{h}} d S_{\mu \nu} D^{\mu} \xi^{\nu}\right)=\frac{1}{8 \pi G_{N}} \int_{V} d S_{\mu} D_{\nu} D^{\mu} \xi^{\nu}
$$

where $V$ is the $(D-1)$-dimensional volume between $S_{h}$ and $S_{\infty}$ so that $\partial V=S_{h} \cup S_{\infty}$. Now we use that for a Killing vector we have $D_{\nu} D^{\mu} \xi^{\nu}=R_{\nu}^{\mu} \xi^{\nu}$ along with the fact that 
the sourced Einstein equation $R_{\mu \nu}=8 \pi G S_{\mu \nu}^{\text {mat }}$ holds everywhere in $V$, as we are away from the black object. We thus have

$$
I_{S_{h}}=I_{S_{\infty}}+\int_{V} d S_{\mu}\left(S^{\mathrm{mat}}\right)_{\nu}^{\mu} \xi^{\nu}
$$

Since we have a static solution we can choose $\xi$ to be the time-translation Killing vector field, i.e. $\xi=\partial / \partial t$. We then compute [16]

$$
I_{S_{h}}=-\frac{1}{8 \pi G_{N}} \int_{S_{h}} d A k^{\nu}\left(D_{\nu} k^{\mu}\right) n_{\mu}=-\frac{\kappa}{8 \pi G_{N}} \int_{S_{h}} d A k^{\mu} n_{\mu}=\frac{\kappa A}{8 \pi G_{N}}=T S .
$$

On the other hand we have asymptotically

$$
I_{S_{\infty}}=-\frac{1}{16 \pi G_{N}} \int_{S_{\infty}} d S_{0 r} \partial_{r} g_{00}=\frac{1}{D-2}\left[(D-3) M-\sum_{a} L_{a} \mathcal{T}_{a}\right]
$$

Here we first used that the non-zero components of $D^{\mu} k^{\nu}$ are $D^{0} k^{r}=-D^{r} k^{0}=\frac{1}{2} \partial_{r} g_{00}$ to leading order, and (B.11), (B.12) are used in the last step. Finally we need to compute the last term in (C.3) which reads

$$
\begin{aligned}
\int_{V} d S_{0}\left(S^{\mathrm{mat}}\right)^{0}{ }_{0} & =-\frac{1}{D-2} \int d^{D-1} x\left[(D-3) T_{00}^{\mathrm{mat}}+\sum_{a} L_{a} T_{a a}^{\mathrm{mat}}\right] \\
& =-\frac{1}{D-2}\left[(D-3) M^{\mathrm{mat}}-\sum_{a} L_{a} \mathcal{T}_{a}^{\mathrm{mat}}\right]
\end{aligned}
$$

where we recall the definition (B.1) of $S_{\mu \nu}$. Putting it all together then yields the Smarr formula

$$
(D-3)\left(M-M^{\text {mat }}\right)=(D-2) T S+\sum_{a} L_{a}\left(\mathcal{T}_{a}-\mathcal{T}_{a}^{\text {mat }}\right)
$$

\section{References}

[1] J. Traschen and D. Fox, "Tension perturbations of black brane spacetimes," gr-qc/0103106.

[2] P. K. Townsend and M. Zamaklar, "The first law of black brane mechanics," Class. Quant. Grav. 18 (2001) 5269-5286, hep-th/0107228.

[3] T. Harmark and N. A. Obers, "New phase diagram for black holes and strings on cylinders," Class. Quantum Grav. 21 (2004) 1709-1724, hep-th/0309116.

[4] B. Kol, E. Sorkin, and T. Piran, "Caged black holes: Black holes in compactified spacetimes I - theory," hep-th/0309190.

[5] R. C. Myers, "Stress tensors and Casimir energies in the AdS/CFT correspondence," Phys. Rev. D60 (1999) 046002, hep-th/9903203. 
[6] A. M. Awad and C. V. Johnson, "Holographic stress tensors for Kerr-AdS black holes," Phys. Rev. D61 (2000) 084025, hep-th/9910040.

[7] A. DeBenedictis and K. S. Viswanathan, "Stress-energy tensors for higher dimensional gravity," hep-th/9911060.

[8] R.-G. Cai and N. Ohta, "Surface counterterms and boundary stress-energy tensors for asymptotically non-anti-de Sitter spaces," Phys. Rev. D62 (2000) 024006, hep-th/9912013.

[9] S. W. Hawking and G. T. Horowitz, "The gravitational Hamiltonian, action, entropy and surface terms," Class. Quant. Grav. 13 (1996) 1487-1498, gr-qc/9501014

[10] J. Traschen, "A positivity theorem for gravitational tension in brane spacetimes," hep-th/0308173.

[11] T. Shiromizu, D. Ida, and S. Tomizawa, "Kinematical bound in asymptotically translationally invariant spacetimes," gr-qc/0309061.

[12] T. Harmark and N. A. Obers, "Black holes on cylinders," JHEP 05 (2002) 032, hep-th/0204047.

T. Harmark and N. A. Obers, "Phase structure of black holes and strings on cylinders," Nucl. Phys. B684 (2004) 183-208, hep-th/0309230

E. Sorkin, B. Kol, and T. Piran, "Caged black holes: Black holes in compactified spacetimes. II: 5d numerical implementation," hep-th/0310096.

H. Kudoh and T. Wiseman, "Properties of Kaluza-Klein black holes," hep-th/0310104.

T. Harmark, "Small black holes on cylinders," hep-th/0310259. To appear in Phys. Rev. D.

E. Sorkin, "A critical dimension in the black-string phase transition," hep-th/0402216.

[13] T. Harmark and N. A. Obers. Work in progress.

H. Elvang, T. Harmark and N. A. Obers. Work in progress.

[14] R. M. Wald, General Relativity. The University of Chicago Press, 1984.

[15] R. Arnowitt, S. Deser, and C. Misner, "The dynamics of General Relativity," in Gravitation: An introduction to current research, L. Witten, ed. New York, Wiley, 1962.

[16] P. K. Townsend, "Black holes," gr-qc/9707012 